\title{
Risk factor for 31-day unplanned readmission to hospital in patients with pulmonary tuberculosis in China
}

Jing Cao MM, MS, Shengpeng Liu, MD, PhD, Juhua Huang, MM, MS.

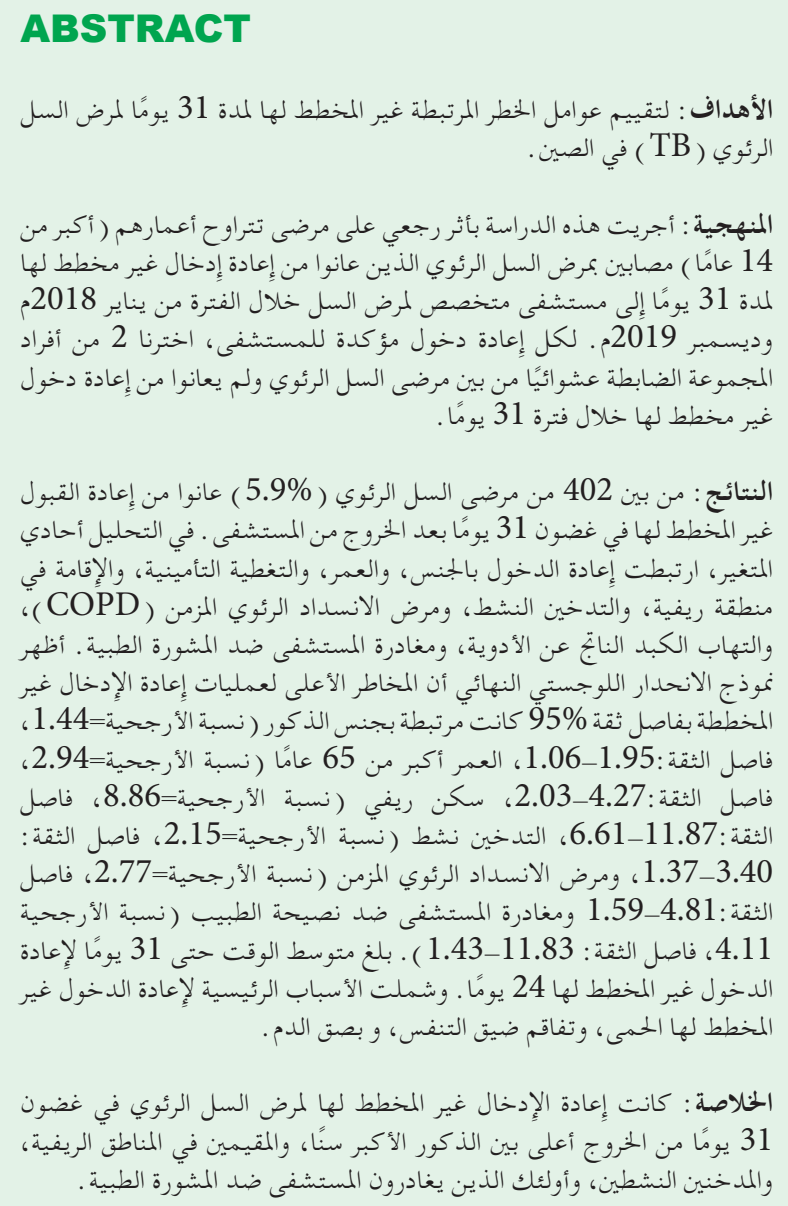

Objectives: To evaluate risk factors associated with 31-day unplanned readmission(s) for pulmonary tuberculosis $(\mathrm{TB})$ in China.

Methods: This retrospective study enrolled patients (age, >14 years) with pulmonary TB who experienced 31-day unplanned readmissions to a specialized hospital for TB between January 2018 and December 2019. For each confirmed readmission, 2 control subjects were randomly selected from among patients with pulmonary
TB but did not experience an unplanned readmission within 31 days.

Results: A total of 402 pulmonary TB patients (5.9\%) experienced unplanned readmission within 31 days after discharge. In univariate analysis, readmission was associated with gender, age, insurance coverage, residing in a rural area, active smoking, chronic obstructive pulmonary disease (COPD), drug-induced hepatitis, and leaving hospital against medical advice. The final logistic regression model revealed that higher risks for unplanned readmissions were associated with male gender (odds ratio [OR] 1.44, [95\% confidence interval (CI) : 1.061.95]), age $>65$ years (OR 2.94, 95\%CI: 2.03-4.27), rural residence (OR 8.86, 95\%CI: 6.61-11.87), active smoking (OR 2.15, 95\% CI 1.37-3.40), COPD (OR 2.77, 95\%CI: 1.59-4.81), and leaving hospital against physician advice (OR 4.11, 95\%CI: 1.43-11.83). The median time to 31-day unplanned readmission was 24 days. Major reasons for unplanned readmission included fever, exacerbation of dyspnea, and hemoptysis.

Conclusion: Unplanned readmission for pulmonary TB within 31 days of discharge was higher among older males residing in rural areas, active smokers, and those leaving hospital against medical advice.

Keywords: pulmonary tuberculosis, unplanned readmission, hospitalization, risk factor

Saudi Med J 2021; Vol. 42 (9): 1017-1023 doi: 10.15537/smj.2021.42.9.20210281

From the Department of Statistical Office, Changsha Central Hospital (Cao), Changsha; from the Pediatric Department, Shenzhen People's Hospital (Lie), Shenzhen; and from the Chronic Disease Prevention and Treatment Department, Loudi Center For Disease Control And Prevention (Huang), Loudi, China.

Received 19th April 2021. Accepted 28th July 2021.

Address correspondence and reprint request to: Dr. Jing Cao, Department of Statistical Office, Changsha Central Hospital, Changsha Central Hospital, Changsha, China.E-mail:523060736@qq.com

ORCID ID: https://orcid.org/0000-0003-4006-9726 
T uberculosis (TB) is one of the most important infectious diseases in the world, and caused by Mycobacterium TB. In accordance with World Health Organization (WHO) report in 2020, the incidence of TB was approximately 10 million (range, 8.9-11.0 million), with 1.4 million deaths worldwide. ${ }^{1}$ In 2020 , the incidence rate of $\mathrm{TB}$ was estimated to be 58 per 100,000 population in China. ${ }^{2}$ Although the prevalence of TB has decreased through 2 decades of effort and short-course strategy programs (such as, directly observed treatment, short course [DOTS]), China still ranks second in terms of the largest burden of TB in the world, which is also associated with enormous economic implications. ${ }^{3}$

Unplanned re-hospitalization within 31 days of discharge refers to a situation in which a patient is readmitted to hospital for the same or related disease within 31 days after initial discharge,and is considered to be a "sentinel event" reflecting poor-quality hospital care. ${ }^{4}$ Moreover, unplanned readmission(s) after hospitalization also impose a burden on patients and health care resources due to high costs. ${ }^{5}$ In 2004 , hospital readmissions in the United States cost the medicare system approximately $\$ 17.5$ billion, accounting for approximately one-fifth of the country's total hospital costs. ${ }^{6}$ A cross-sectional study reported a hospital readmission rate of $5.9 \%$, which varied among hospitals, ranging from $9.8 \%$ to $17.0 \%$ in France. ${ }^{7}$ The readmission rates continue to rise, causing widespread concern across countries. The United States and China announced in 2007 and 2011, respectively, that early unplanned readmission should be used as an important indicator for evaluating medical quality. ${ }^{8,9}$

Considering the additional costs that unplanned readmission impose to the health system, correlational research may improve our clinical decision-making on unplanned hospitalization. A systematic study in 2020, chronic obstructive pulmonary disease (COPD)-related readmission rates have differences in 30 countries, risk factors and the local healthcare environment should be interventions. ${ }^{10}$ According to a 3-year study carried out in Iran, a common cause of early readmission were druginduced hepatitis, dyspnea. ${ }^{11}$ In addition, comorbidities and socioeconomic factors were also considered to be associated with readmission. ${ }^{12,13}$ So far, readmissions for

Disclosure. Authors have no conflict of interests, and the work was not supported or funded by any drug company. major disease and surgical diagnoses have been studied, but the risk factors for early unplanned readmission among patients with pulmonary TB without research. Thus, this study aimed to investigate risk factors associated with 31-day unplanned readmission of patients previously discharged with pulmonary $\mathrm{TB}$ in China.

Methods. The present investigation was a retrospective analysis of adult (age $>14$ years) patients with confirmed pulmonary TB admitted to Changsha Central Hospital, Hunan, China between January 2018 and December 2019. Patient diagnoses were coded according to the WHO's International Classification of Diseases (10th Revision), and matched to cases according to the principal diagnosis. Readmission data were collected from the electronic medical record monitoring database, and were verified to confirm whether they documented unplanned readmission(s) within 31 days of admission for TB. The unplanned readmission group included patients who were readmitted for TB related diseases within 31 days after discharge. In contrast, patients lack of readmission information; HIV positive patients; scheduled for readmission; readmitted for a non-TBrelated condition were excluded. For each confirmed readmission, 2 control subjects were randomly selected from among patients with pulmonary $\mathrm{TB}$ who did not experience unplanned readmission within 31 days. Demographic information and clinical characteristics of the patients, including gender, age, TB type, medical comorbidities, and so on, were obtained from the hospital's electronic record systems.

Direct sputum smear method was used for sputum smear (Ziehl-Neelson), then was smeared and observed with a microscope. The operation is strictly in accordance with the "Manual of Standardized Operation and Quality Assurance for Sputum Smear Microscopy" (Olympus CX31, Japan) biological microscope was used for the instrument, and anti-acid staining is Zhuhai BASO Company (China). Detection standard for positive sputum smear microscopy: if the number of acid-fast bacilli in 100 visual fields is 3-9, it indicates a positive result for TB bacilli.

PubMed database was used for identification and retrieval the article. This study was approved by the Medical Ethics Committee, Changsha Central Hospital and in accordance with the Helsinki Declaration.

Statistical analysis. All statistical analyses were performed using IBM SPSS Statistics for Windows, version 25.0 (IBM Corp., Armonk, N.Y., USA). Univariate analysis (a simple binary logistic regression) was used to identify the potential factors associated with 
readmission (patients unplanned readmitted $\leq 31$ days and no unplanned readmission $\leq 31$ days). Variables whose value of $p<0.05$ were included in the multiple analysis (binary logistic regression model [multiple]) to adjust for possible biases. Variables were selected with the enter method, and the goodness of fit was evaluated by the Hosmer-Lemeshow test. Odds ratios (OR) and their respective $95 \%$ confidence intervals (95\% CI) were estimated. Reference categories were assigned $\mathrm{OR}=1$. Statistical significance was based on 2-tailed $p<0.05$.

Results. Between January 2018 and December 2019, 6806 patients with pulmonary TB were admitted to the Changsha Central Hospital, Changsha, China of which 402 (5.9\%) were unplanned readmissions after discharge. Accordingly, a total of 804 controls were randomly selected from among patients who had not experienced unplanned readmission. As such, 1206 patients (unplanned readmission $\leq 31$ days [ $\mathrm{n}=402$ ], non-unplanned readmission $\leq 31$ days $[\mathrm{n}=804]$ ) were fit into the study. The mean ( \pm standard deviation [SD]) age was $48.20 \pm 18.98$ years; 786 were male and 420 were female.

The median time to 31-day re-hospitalization was 24 days, with a range of $0-31$ days. Four patients were readmitted on the day of discharge, comprising of $1.0 \%$ of the total readmission rate, $104(25.9 \%)$ were readmitted to hospital within 1 to 15 days after discharge, and $298(74.1 \%)$ were readmitted 16 to 31 days after initial discharge (Figure 1).

Univariate analyses revealed that patients who experienced unplanned readmission differed significantly from non-readmitted patients in terms of gender, age, insurance coverage, rural versus urban residency, active smoking, COPD, drug- induced hepatitis, and leaving hospital against medical advice (Table 1).

Multivariate analysis included all significant variables from univariate analysis $(p<0.05)$. In the final model showed that male gender, age $>65$ years, rural residence, active smoking, COPD, and leaving hospital against medical advice retained significance (Table 1).

Discussion. The unplanned readmission rate for a 2-year period in this study was $5.91 \%$, which as lower than the rates reported by Silva and Qayed. ${ }^{14,15}$ The median time to 31-day unplanned readmission was 24 days, and $>70 \%$ of patients were readmitted between 16 and 31 days after discharge.

Unplanned readmission for pulmonary $\mathrm{TB}$ was higher in males, which is usually the case with $\mathrm{TB}$ infection. The readmission rate according to gender was higher in some studies, but only one study reported a direct relationship. ${ }^{16}$ Based on univariate and multivariate regression analyses, our study also confirmed that males were readmitted more often. Regarding the place of residence, patients residing in rural areas experienced higher readmission rates than those in urban areas, which is different from the results reported in a study from Brazil. ${ }^{14}$ This difference may have several explanations. First, the prevalence of TB is higher in rural versus urban areas of China, ${ }^{17}$ and those residing in rural areas are more affected by poor socioeconomic conditions. These patients often delay their medical treatment after falling ill, which leads to them becoming a new source of infection and infecting

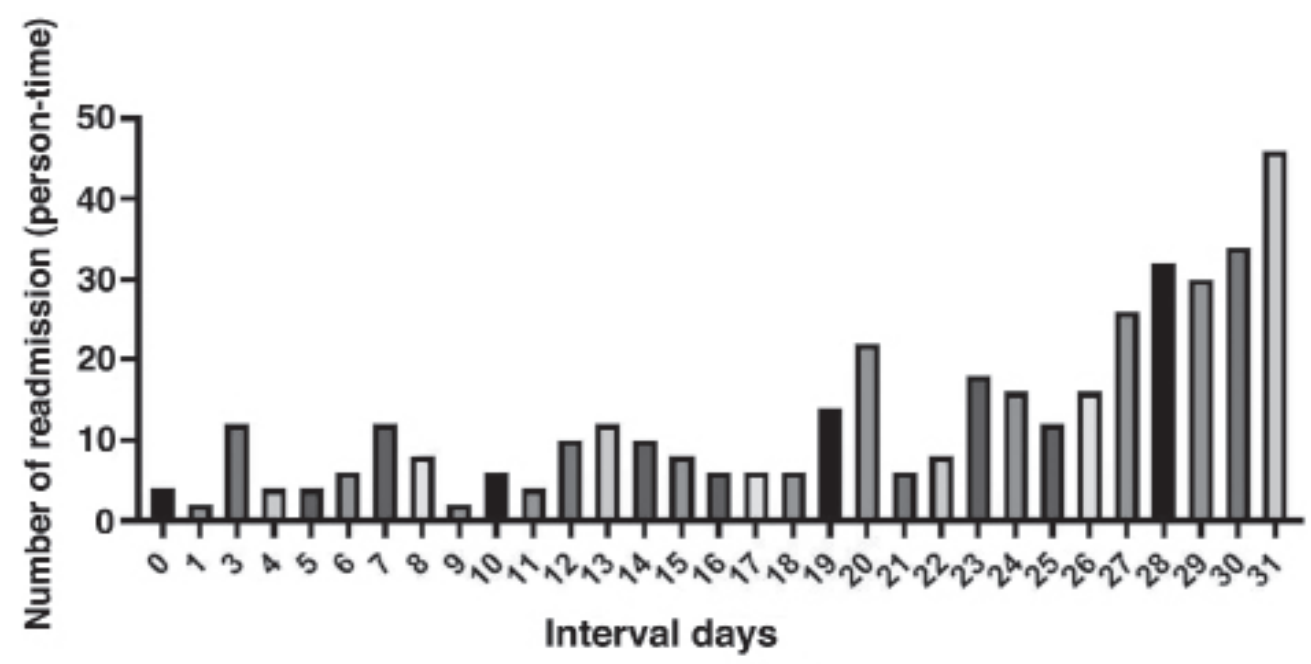

Figure 1 - Discharge-readmission days distribution chart. 
Unplanned readmission of PTB patients... Cao et al

Table 1 - Unplanned readmission $\leq 31$ days risk factors for pulmonary tuberculosis patients.

\begin{tabular}{|c|c|c|c|c|c|c|}
\hline \multirow{2}{*}{ Variables } & \multirow{2}{*}{$\begin{array}{l}\text { No unplanned } \\
\text { readmission } \leq 31 \text { days } \\
(\mathrm{n}=804)\end{array}$} & \multirow{2}{*}{$\begin{array}{l}\text { Unplanned } \\
\text { readmission } \leq 31 \\
\text { days }(\mathrm{n}=402)\end{array}$} & \multicolumn{2}{|c|}{ Univariate analysis } & \multicolumn{2}{|c|}{ Multiple analysis } \\
\hline & & & OR $(95 \% \mathrm{CI})$ & $P$-value & OR $(95 \% \mathrm{CI})$ & $P$-value \\
\hline \multicolumn{7}{|l|}{ Gender } \\
\hline Male & $506(62.9)$ & $280(69.7)$ & $1.35(1.05-1.75)$ & 0.021 & $1.44(1.06-1.95)$ & 0.02 \\
\hline Female & $298(371)$ & $122(30.35)$ & 1 & & 1 & \\
\hline \multicolumn{7}{|l|}{ Age group } \\
\hline $15-44$ & $300(37.3)$ & $126(31.3)$ & 1 & & 1 & \\
\hline $45-65$ & $361(44.9)$ & $133(33.1)$ & $0.88(0.66-1.17)$ & 0.372 & $1.08(0.77-1.51)$ & 0.674 \\
\hline$>65$ & $143(17.8)$ & $143(35.6)$ & $2.38(1.74-3.25)$ & $<0.001$ & $2.94(2.03-4.27)$ & $<0.001$ \\
\hline \multicolumn{7}{|l|}{ Insurance type } \\
\hline $\begin{array}{l}\text { Medical insurance for urban } \\
\text { workers }\end{array}$ & $192(23.9)$ & $88(21.89)$ & $0.29(0.14-0.62)$ & 0.001 & $0.53(0.22-1.27)$ & 0.155 \\
\hline $\begin{array}{l}\text { Medical insurance for urban } \\
\text { residents }\end{array}$ & $254(31.6)$ & $95(23.6)$ & $0.24(0.11 \sim 0.51)$ & 0.002 & $0.42(0.18 \sim 1.01)$ & 0.051 \\
\hline New rural cooperative medical & $319(39.7)$ & $180(44.8)$ & $0.36(0.17-0.75)$ & $<0.001$ & $0.53(0.23 \sim 1.25)$ & 0.145 \\
\hline Other commercial insurance & $27(3.4)$ & $20(5.0)$ & $0.47(0.19-1.18)$ & 0.007 & $0.68(0.24-1.99)$ & 0.484 \\
\hline Self-paying & $12(1.5)$ & $19(4.7)$ & 1 & & 1 & \\
\hline \multicolumn{7}{|l|}{ Marital status } \\
\hline Single & $95(11.8)$ & $40(10.0)$ & $1.21(0.82-1.79)$ & 0.333 & $\mathrm{~N} / \mathrm{I}$ & \\
\hline Married & $709(88.2)$ & $362(90.1)$ & 1 & & & \\
\hline \multicolumn{7}{|l|}{ Residency } \\
\hline Rural & $169(21.0)$ & $283(70.4)$ & $8.94(6.80-11.74)$ & $<0.001$ & $8.86(6.61-11.87)$ & $<0.001$ \\
\hline Urban & $635(79.0)$ & $119(29.6)$ & 1 & & 1 & \\
\hline \multicolumn{7}{|l|}{ Active smoking } \\
\hline Yes & $57(7.0)$ & $61(15.2)$ & $2.34(1.60-3.44)$ & $<0.001$ & $2.15(1.37-3.40)$ & 0.001 \\
\hline No & $747(92.9)$ & $341(84.8)$ & 1 & & 1 & \\
\hline \multicolumn{7}{|l|}{ Patient tuberculosis type } \\
\hline Smear negative & $179(22.3)$ & $102(25.4)$ & $0.84(0.64-1.11)$ & 0.229 & $\mathrm{~N} / \mathrm{I}$ & \\
\hline Smear positive & $625(77.7)$ & $300(74.6)$ & 1 & & & \\
\hline \multicolumn{7}{|l|}{ Diabetes } \\
\hline Yes & $48(6.0)$ & $35(8.7)$ & $1.52(0.96-2.36)$ & 0.078 & $N / I$ & \\
\hline No & $756(94.0)$ & $367(91.3)$ & 1 & & & \\
\hline \multicolumn{7}{|l|}{ Alcohol abuse } \\
\hline Yes & $46(5.7)$ & $30(7.5)$ & $1.33(0.83-2.14)$ & 0.242 & $\mathrm{~N} / \mathrm{I}$ & \\
\hline No & $758(94.3)$ & $372(92.5)$ & 1 & & & \\
\hline \multicolumn{7}{|l|}{$C O P D$} \\
\hline Yes & $27(3.4)$ & $47(11.7)$ & $3.81(2.34-6.22)$ & $<0.001$ & $2.77(1.59-4.81)$ & $<0.001$ \\
\hline No & 777 (96.6) & $355(88.3)$ & 1 & & 1 & \\
\hline \multicolumn{7}{|l|}{ Drug-induced hepatitis } \\
\hline Yes & $41(5.1)$ & $33(8.2)$ & $1.66(1.04-2.68)$ & 0.036 & $1.22(0.69-2.17)$ & 0.492 \\
\hline No & $763(94.9)$ & $369(91.8)$ & 1 & & 1 & \\
\hline \multicolumn{7}{|l|}{ Hypertension } \\
\hline Yes & $48(6.0)$ & $32(8.0)$ & $1.36(0.86-2.17)$ & 0.191 & $\mathrm{~N} / \mathrm{I}$ & \\
\hline No & $756(94.03)$ & $370(92.0)$ & 1 & & & \\
\hline \multicolumn{7}{|l|}{ Departure from the hospital way } \\
\hline $\begin{array}{l}\text { leaving hospital against } \\
\text { medical advice }\end{array}$ & $8(36.4)$ & $14(63.6)$ & $3.59(1.49-8.63)$ & 0.004 & $4.11(1.43-11.83)$ & 0.009 \\
\hline $\begin{array}{l}\text { leaving hospital with medical } \\
\text { advice }\end{array}$ & $796(67.2)$ & $388(32.8)$ & 1 & & 1 & \\
\hline \multicolumn{7}{|l|}{ Disease type } \\
\hline Retreatment & $154(19.1)$ & $62(15.4)$ & $1.29(0.93-1.78)$ & 0.124 & $\mathrm{~N} / \mathrm{I}$ & \\
\hline New case & $650(80.9)$ & $340(84.6)$ & 1 & & & \\
\hline
\end{tabular}


other susceptible groups. ${ }^{18}$ Furthermore, poverty leads to low protein intake, which negatively affects the body's immune system. Poverty is also associated with lower education levels, which indirectly reduces an individual's ability to prevent TB. ${ }^{19-21}$ Therefore, TB health education in the future should remain focused on rural areas.

Unplanned readmission of $\mathrm{TB}$ patients was associated with age $>65$ years, similar to the findings reported by Anaam. ${ }^{16}$ But, this results differ from another study, in which age was not confirmed as a risk factor for hospital readmission among TB patients. ${ }^{11}$ The association between readmission and age can be explained by decreased immunity, comorbidity, and concomitant diseases. Studies investigating hospital readmission among $\mathrm{TB}$ patients report different risk factors; however, all depend on comorbidity. ${ }^{11,22}$ Results of our study demonstrated that COPD is one of the risk factors for increased unplanned readmission. A study by Bhatt et $\mathrm{al},{ }^{23}$ documented in middle-aged and older patients with COPD have a higher risk for re-hospitalization. A follow-up study reported that COPD patients with a history of pulmonary TB were hospitalized more often and experienced lower long-term survival rates compared to those without a history of TB. ${ }^{24}$ Chronic obstructive pulmonary disease is not only a important risk factor for $\mathrm{TB}$, but is also one of the important causes of Mycobacterium TB drug resistance. ${ }^{25}$ Additionally, the readmission rate of smoking population was significantly higher than that of non-smoking population, and tobacco has an impact on both TB/COPD morbidity and mortality. ${ }^{26,27}$ Various studies have verified that smoking is a risk factor for TB and COPD. However, results of our study do no brief for the formulation that smoking causes additional mortality risk in those with pulmonary $\mathrm{TB},{ }^{28}$ but do suggest that smoking is a risk factor for unplanned readmission.

The unplanned readmission rate for patients who leave the hospital against physician/medical advice was also higher. These patients were discharged from the hospital at their own request due to financial or personal reasons without completing treatment or meeting discharge indications. Existing studies have indicated that treatment failure is, in large part, attributable to antimicrobial-resistant pulmonary TB.$^{29} \mathrm{~A}$ nested casecontrol study documented that properly managing patients who experience their first TB infection by encouraging strict adherence to medication do not fail treatment, and can be rescued from relapse. ${ }^{30}$ To reduce the rate of patients leaving hospital without medical advice, we need to make joint efforts at the national and hospital levels, such as strengthening the medical system, reducing the proportion of patients paying out-of-pocket expenses, and improving the treatment capability of hospitals treating severely ill patients. According to the Chinese hospital monitoring system readmission reporting requirements, we subcategorized the time interval into 3 groups (according to time of first discharge and interval of readmission). In the first group (readmission on the same day), we evaluated patients who were readmitted on the day of discharged patients within the same days, in which sudden relapse occurred on the day of discharge, including dizziness, vomiting, and pneumothorax. Treatment refusal and treatment failure were the main causes of late readmission (such as, 16 to 31 days after discharge). Exacerbation of dyspnea and fever is a common objective reason for both early and late readmission, followed by drug side-effects and hemoptysis.

The present study had several strengths. Our hospital is a specialized tuberculosis hospital with a relatively stable patient source, so a large sample size can be obtained in this study. The readmission of COPD and asthma has been studied by some scholars, ${ }^{31,32,33}$ but only a few studies have looked at readmission rates for TB, and there is a lack of research on unplanned readmission in patients with pulmonary TB. This study analyzed the influencing factors of unplanned readmission of $\mathrm{TB}$ patients, which could help to identify and intervene the high-risk groups of unplanned readmission. Meanwhile, this study also provides a theoretical basis for subsequent prospective studies of unplanned readmission to TB.

Study limitations. First, was it is a single-center, retrospective design, which may have hindered some analyses. We advise that early unplanned readmission is an critical parameter to monitor in TB programs, and prospective studies should be initiated in the future. Second, this survey is a single-center study. The HIV infection rate in this province is relatively low, and TB patients will be transferred to the provincial infectious disease hospital immediately once they are diagnosed with AIDS, so the readmission information of such patients cannot be collected. Third, although a few behaviors and socioeconomic characteristics of patients were collected, this retrospective study was unable to obtain more information relevant to patient readmission, such as socioeconomic status, body mass index, pharmacological intake, and education.

In conclusion, the unplanned readmission rate of pulmonary $\mathrm{TB}$ in our study was lower compared to similar studies. The most common reasons for unplanned readmission to our hospital included fever, exacerbation of dyspnea, and drug side effects. We conclude that the 
unplanned readmission within 31 days of pulmonary $\mathrm{TB}$ was higher in older males residing in rural areas, and among active smokers, and those leaving the hospital against medical advice. The existence of these factors reminds the medical staff to attach great importance to the newly hospitalized patients with these factors. Some of these factors can be changed by intervention, indicating that continuous health management and regular treatment can be achieved for patients with chronic comorbidities, smoking cessation, strengthen health education in rural areas, and reducing the ratio of out-of-pocket patient expenses, may help decrease unplanned readmission rates within 31 days for pulmonary TB.

Acknowledgment. The authors gratefully Editage (www.editage.com) for English language editing.

\section{References}

1. World Health Organization. Global tuberculosis report 2020. (Updated 2020 October 15. Accessed 2020 October 22). Available from: https://www.who.int/publications/i/ item/9789240013131

2. Yang T, Chen T, Che Y, Chen Q, Bo D. Factors associated with catastrophic total costs due to tuberculosis under a designated hospital service model: a cross-sectional study in China. BMC Public Health 2020; 20: 1009.

3. Cui X, Gao L, Cao B. The management of latent TB infection in China needs to explore a new approach suitable for high-burden countries. Chinese Journal of Antituberculosis 2020; 42: 390.

4. Duggal R, Zhang Y, Diana ML. The association between hospital ACO participation and readmission rates. J Healthc Manag 2018; 63: e100-e114.

5. Finlayson K, Chang AM, Courtney MD, Edwards HE, Parker AW, Hamilton K, et al. Transitional care interventions reduce unplanned hospital readmissions in high-risk older adults. BMC Health Serv Res 2018; 18: 956.

6. Daras LC, Deutsch A, Ingber MJ, Hefele JG, Perloff J. Inpatient rehabilitation facilities' hospital readmission rates for medicare beneficiaries treated following a stroke. Top Stroke Rehabil 2021; 28: 61-71.

7. Lefèvre JH, Reboul-Marty J, de Vaugrigneuse S, Zeitoun JD. Readmissions after surgery: a french nationwide cross-sectional study of 1,686,602 procedures performed in 2010. World J Surg 2017; 41: 31-38.

8. Hoffman H, Protas M, Chin LS. A nationwide analysis of 30-day and 90-day readmissions after elective cerebral aneurysm clipping in the United States: causes, predictors, and trends. World Neurosurg 2019; 128: e873-e883.

9. Wang Xiaoying, Wang Xiaoxia, Song Ying. Study on unplanned readmission patients in stomatological hospital within 31 days. China Health Statistics 2017; 34: 293-295.

10. Njoku CM, Alqahtani JS, Wimmer BC, Peterson GM, Kinsman L, Hurst JR, et al. Risk factors and associated outcomes of hospital readmission in COPD: A systematic review. Respir Med 2020; 173: 105988.
11. Shamaei M, Samiei-Nejad M, Nadernejad M, Baghaei P. Risk factors for readmission to hospital in patients with tuberculosis in Tehran, Iran: three-year surveillance. Int J STD AIDS 2017; 28: 1169-1174.

12. Rørth R, Fosbol EL, Mogensen UM, Kragholm K, Numé AK, Gislason GH, et al. Employment status at time of first hospitalization for heart failure is associated with a higher risk of death and rehospitalization for heart failure. Eur J Heart Fail 2018; 20: 240-247.

13. Scaria E, Powell WR, Birstler J, Alagoz O, Shirley D, Kind AJH, et al. Neighborhood disadvantage and 30-day readmission risk following clostridioides difficile infection hospitalization. BMC Infect Dis 2020; 20: 762.

14. Silva TC, Matsuoka PDFS, Aquino DMC, Caldas AJM. Factors associated with tuberculosis retreatment in priority districts of Maranhão, Brazil. Cien Saude Colet 2017; 22: 4095-4104.

15. Qayed E, Muftah M. Frequency of hospital readmission and care fragmentation in gastroparesis: A nationwide analysis. World J Gastrointest Endosc 2018; 10: 200-209.

16. Anaam MS, Alrasheedy AA, Alsahali S, Alfadly SO, Aldhubhani AH. Rate and risk factors of recurrent tuberculosis in Yemen: a 5-year prospective study. Infect Dis (Lond) 2020; 52: 161-169.

17. Zhao F, Du X, Li T, Wang LX, Zhang H, Liu JJ, et al. Epidemic trend and prediction of tuberculosis in China based on WHO public database. Journal of Clinical Drug Therapy 2018: 16: $1-3$.

18. Shen XB, Kang LL, Zhou YZ,Qu L, Si ZL. Comparison of tuberculosis incidence and molecular epidemiological characteristics between urban and rural areas in northern Guizhou. Practical Preventive Medicine 2019; 26: 321-323.

19. Cheng J, Sun YN, Zhang CY, Yu YL, Tang LH, Peng H, et al. Incidence and risk factors of tuberculosis among the elderly population in China: a prospective cohort study. Infect Dis Poverty 2020; 9: 13.

20. Tegegne BS, Mengesha MM, Teferra AA, Awoke MA, Habtewold TD. Association between diabetes mellitus and multi-drug-resistant tuberculosis: evidence from a systematic review and meta-analysis. Syst Rev 2018; 7: 161.

21. Sürücüoğlu S. Seyahat ile ilişkili tüberküloz riski [Risk of travel associated tuberculosis]. Mikrobiyol Bul 2018; 52: 96-107.

22. Pradipta IS, Van't Boveneind-Vrubleuskaya N, Akkerman OW, Alffenaar JC, Hak E. Treatment outcomes of drug-resistant tuberculosis in the Netherlands, 2005-2015. Antimicrob Resist Infect Control 2019; 8: 115.

23. Bhatt SP, Balte PP, Schwartz JE, Cassano PA, Couper D, Jacobs DR Jr, et al. Discriminative accuracy of FEV1:FVC thresholds for COPD-related hospitalization and mortality. JAMA 2019; 321: 2438-2447.

24. Yakar HI, Gunen H, Pehlivan E, Aydogan S. The role of tuberculosis in COPD. Int J Chron Obstruct Pulmon Dis 2017; 12: 323-329.

25. Tacconelli E, Carrara E, Savoldi A, Harbarth S, Mendelson M, Monnet DL, et al. Discovery, research, and development of new antibiotics: the WHO priority list of antibiotic-resistant bacteria and tuberculosis. Lancet Infect Dis 2018; 18: 318 -327.

26. Perriot J, Underner M, Peiffer G, Flaudias V. Stratégie et modalités d'aide à l'arrêt du tabac chez les fumeurs tuberculeux [Strategy and stopping smoking interventions in smokers with tuberculosis]. Rev Med Liege 2020; 75: 100-104. 
27. Byrne AL, Marais BJ, Mitnick CD, Lecca L, Marks GB. Tuberculosis and chronic respiratory disease: a systematic review. Int J Infect Dis 2015; 32: 138-146.

28. Perriot J, Underner M, Peiffer G. Le tabagisme et l'aide à l'arrêt du tabac des patients atteints de tuberculose [Tobacco smoking and stopping smoking interventions in patients with TB]. Rev Pneumol Clin 2018; 74: 391-399.

29. Abdelbary BE, Garcia-Viveros M, Ramirez-Oropesa H, Rahbar $\mathrm{MH}$, Restrepo BI. Predicting treatment failure, death and drug resistance using a computed risk score among newly diagnosed TB patients in Tamaulipas, Mexico. Epidemiol Infect 2017; 145: 3020-3034.

30. Bestrashniy JRBM, Nguyen VN, Nguyen TL, Pham TL, Nguyen TA, Pham DC, et al. Recurrence of tuberculosis among patients following treatment completion in eight provinces of Vietnam: A nested case-control study. Int J Infect Dis 2018; 74: 31-37.
31. Wu CW, Lan CC, Hsieh PC, Tzeng IS, Wu YK. Role of peripheral eosinophilia in acute exacerbation of chronic obstructive pulmonary disease. World J Clin Cases 2020 6; 8: 2727-2737.

32. Breyer-Kohansal R, Hartl S, Breyer MK, Schrott A, Studnicka M, Neunhäuserer D, Fülöp G, Burghuber OC. The European COPD audit: Adherence to guidelines, readmission risk and hospital care for acute exacerbations in Austria. Wien Klin Wochenschr 2019; 131: 97-103.

33. Sun W, Pan L, Zhang W. Risk factors for readmission of children hospitalized with acute asthma attacks in South China. J Asthma 2021; 58: 438-447. 